\title{
QUEEN'S
UNIVERSITY
BELFAST
}

\section{Assessing Current HMI Designs and Exploring Al Potential for Future Air-Defence System Development}

Gibson, Z., Butterfield, J., Ferguson, S., Rafferty, K., Yu, W., \& Casement, A. (2020). Assessing Current HMI Designs and Exploring Al Potential for Future Air-Defence System Development. In S. Yamamoto, \& H. Mori (Eds.), International Conference on Human-Computer Interaction: Human Interface and the Management of Information (Vol. 2, pp. 305-323). (Lecture Notes in Computer Science; Vol. 12185). Springer. https://doi.org/10.1007/978-3-030-50017-7_22

Published in:

International Conference on Human-Computer Interaction

Document Version:

Peer reviewed version

Queen's University Belfast - Research Portal:

Link to publication record in Queen's University Belfast Research Portal

Publisher rights

Copyright 2020 Springer. This work is made available online in accordance with the publisher's policies. Please refer to any applicable terms of use of the publisher.

\section{General rights}

Copyright for the publications made accessible via the Queen's University Belfast Research Portal is retained by the author(s) and / or other copyright owners and it is a condition of accessing these publications that users recognise and abide by the legal requirements associated with these rights.

Take down policy

The Research Portal is Queen's institutional repository that provides access to Queen's research output. Every effort has been made to ensure that content in the Research Portal does not infringe any person's rights, or applicable UK laws. If you discover content in the Research Portal that you believe breaches copyright or violates any law, please contact openaccess@qub.ac.uk. 


\title{
Assessing Current HMI Designs and Exploring AI Potential for Future Air-Defence System Development
}

\author{
Zara Gibson $^{1[0000-0001-6907-3644]}$, Joseph Butterfield ${ }^{1}$, Robin Stuart Ferguson ${ }^{1}$, Karen Raf- \\ ferty $^{1}$, Wai $\mathrm{Yu}^{2}$ and Alfred Casement ${ }^{2}$ \\ ${ }^{1}$ Queen's University Belfast, University Road, Belfast, Northern Ireland \\ 2 Thales UK, Alanbrooke Road, Belfast, Northern Ireland \\ z.gibson@qub.ac.uk
}

\begin{abstract}
Designing human-machine interfaces (HMIs) for defence systems (such as vehicle-based weapon terminals) faces numerous challenges such as limited workspace, operation in extreme environments and essential protective gear for operators and equipment. This paper presents a study designed to investigate the HMI and teaming for ground-based air defence systems and reports the findings. The objective of the first stage of the study is to assess the usability, satisfaction, ease of use and design of the current systems to identify avenues for improvement in future system development. The main findings confirm some of the issues identified in the early human factors analysis, such as the lack of space, task complexity and the level of expertise required for efficient system operation The reported findings do not just help identify the areas for improvement in the HMI, but also identify tasks that could be assisted by Artificial Intelligence (AI) integrated within current system functions, to improve human performance whilst also reducing cognitive load, errors and the risk of poor decision-making.
\end{abstract}

Keywords: Ergonomics, Human-machine interface, Military, Artificial intelligence.

\section{Introduction}

Recent technological advances in military system development, especially in sensing and networking, have resulted in increased informational processing demands on operators. These operators are often already experiencing high physical and cognitive load, due to the criticality of accurate decision-making. Resultantly, soldiers at all levels are now faced with larger amounts of information in conjunction with their traditional military role requirements. Junior and non-commissioned officers are assigned greater amounts of information, previously only accessible to higher-level commanders [1].

This additional sensory data aims to improve situational awareness, preparedness and assist in making rapid, coordinated and critical battlefield decisions. The armed forces no longer fight as individuals but as a collective system. As a result, the communications and command systems are vital [2], as effective communications allow armies to complete the 'observe-orient-decide-act' cycle significantly faster. Thereby, complex vehicle systems are required to manage this information. 
Unfortunately, tied closely to this increased flow of information, is the rising concern over how the crew will process and use this information effectively in battle scenarios. The risk of information overload is very real for the modern soldier. Due to the lack of time to make decisions and the increased lethality of modern weaponry, the cognitive demands and stress in modern military operations have increased [3][4]. Elevated stress and higher cognitive demands are linked to an increased likelihood of errors [5], with approximately $80 \%$ of military accidents caused by human error [6]. As a result, the human element of these vehicle electronics needs to be considered moving forwards [2] as technological readiness is not enough to wage war and the cognitive readiness of military personnel is key [7].

Effective human-machine interface (HMI) design can be one approach; efficient HMIs use less attentional resources, enabling more efficient information processing [8]. Using salient stimuli can promote faster and more effortless information processing ([9] provides a review). Salient visual designs can direct visual-spatial attention toward critical visual notifications, reducing visual search time and sparing attentional resources [8]. This shows exactly how important the design of the interface is in enabling better human performance. Ineffective HMIs can cause information overload, leading to erroneous decisions. Understanding the impact of existing (and next-generation) HMI designs in conveying multi-source information, is essential to balance information content, and quantity, with user cognitive limitations to improve performance.

Additionally, there is potential to utilise the technological advances in artificial intelligence (AI; such as automation and intelligent agents) to provide the military with a tactical and cognitive advantage. In a military scenario, AI could increase autonomy, sophistication and dispersion of weapons systems and personnel [10]. A challenge, however, is a lack of theoretical understanding of how humans interact with machines in work contexts e.g. in terms of risk management and resiliency [11][12][13]. In the military, decisions have to be made within tight time schedules, often with incomplete information. Furthermore, mission complexity is outpacing the ability to manage disruptions, which calls for systemic approaches that span technology, human, and mission space [14]. New situational complexity is likely to overload team cognitive resources [15]. As the requirements of systems and their operators are expanded, the tasks approach the operator's response capacity limit; once the limit is reached and exceeded, the system is susceptible to failure [16]. As a result, improved HMIs coupled with AI (to augment human decision-making in complex, high load scenarios) can reduce operational risks associated with poor decision-making.

\subsection{Current study}

This paper aims to apply a questionnaire battery method used in previous work [17] to assess user opinions on the design of two variants of a Thales vehicle-based weapon system; a Light Armored Vehicle (LAV) and Heavy Armored Vehicle (HAV). The vehicles are operated by three crew members; a Commander, Operator and Driver. The Commander Console for the LAV (Weapon Terminal- WT) is an updated version of the HAV Tactical Command Console (TCC). The Operator Control Console (OCC) is the same in both and comprises a display unit and weapon system control panel. 
This study was designed to investigate the HMI and teaming for these ground-based air-defence vehicles. The objective was to assess the usability, satisfaction, ease of use, workload and design (from both a physical and cognitive perspective) of the current systems to identify avenues for improvement in future system development. To achieve this, several standardized questionnaires were compiled into a question battery for current users to complete. Firstly, the questionnaires were screened to extract relevant questions to avoid question repetition and to compress the battery to a more manageable size for participants. Additional questions of interest were included to supplement the questionnaires and answer specific design questions such as examining the respective support the interfaces provided in support of mission-critical tasks. The subscales and individual items were extracted to provide insights on key constructs of the system design such as usability, usefulness, satisfaction etc. as well as the workload associated with specific mission tasks. Additionally, the scope for incorporating intelligent elements in the information displays and controls is inferred from the outputs. Table 1 below details the vehicle weapon systems aspects that the questionnaires examined.

Table 1. Components measured by the questionnaires used in this study*

\begin{tabular}{|l|c|c|c|c|c|c|c|c|}
\hline & \multicolumn{3}{|c|}{ Physical Ergonomics } & \multicolumn{3}{c|}{ Cognitive Ergonomics } \\
\hline & DMQ & NMQ & BPDQ & $\begin{array}{l}\text { Add. } \\
\text { Q's }\end{array}$ & USE & QUIS & $\begin{array}{l}\text { NASA- } \\
\text { TLX }\end{array}$ & $\begin{array}{l}\text { Add. } \\
\text { Q's }\end{array}$ \\
\hline Risk of injury & $\checkmark$ & & & & & & & \\
\hline $\begin{array}{l}\text { Frequency of } \\
\text { bad posture }\end{array}$ & $\checkmark$ & & & & & & & \\
\hline Recent injuries & & $\checkmark$ & & & & & & \\
\hline $\begin{array}{l}\text { End of shift dis- } \\
\text { comfort }\end{array}$ & & & $\checkmark$ & & & & & \\
\hline Visibility & & & & $\checkmark$ & & & & \\
\hline Space & $\checkmark$ & & & $\checkmark$ & & & & \\
\hline Usefulness & & & & & $\checkmark$ & & & \\
\hline Ease of use & & & & & $\checkmark$ & $\checkmark$ & $\checkmark$ & \\
\hline Ease of learning & & & & & $\checkmark$ & $\checkmark$ & & \\
\hline Satisfaction & & & & & $\checkmark$ & & & \\
\hline Screen & & & & & & $\checkmark$ & & \\
\hline $\begin{array}{l}\text { Information } \\
\text { presentation }\end{array}$ & & & & & & $\checkmark$ & & \\
\hline $\begin{array}{l}\text { System capabili- } \\
\text { ties }\end{array}$ & & & & & & $\checkmark$ & & \\
\hline Level of control & & & & & & $\checkmark$ & & \\
\hline System logic & & & & & & $\checkmark$ & & \\
\hline Task demands & & & & & & & $\checkmark$ & \\
\hline Performance & & & & & & & $\checkmark$ & \\
\hline Frustration & & & & & & & $\checkmark$ & \\
\hline Effectiveness & & & & & & & & $\checkmark$ \\
\hline
\end{tabular}

*DMQ- Dutch Musculoskeletal Questionnaire; NMQ- Nordic Musculoskeletal Questionnaire, BDPQ- Body Part Discomfort Questionnaire, USE- Usefulness, Satisfaction and Ease of Use, QUIS- Questionnaire for User Interface Satisfaction, NASA-TLX- Task Load Index 


\section{Method}

\subsection{Participants}

Thirty-seven participants completed the thirty-minute questionnaire. Twenty-seven (4 female) completed the HAV and ten completed the LAV (all male). HAV users were UK participants recruited from the Ministry of Defence and LAV users were overseas Asian participants recruited via system trainers from the overseas bases. Some users had experience of the both the WT/TCC and OCC so completed all parts, resultantly, sixteen HAV users completed the Commander section and eighteen completed the Operator section of the questionnaire. Both sections were completed by all the LAV users. The experience of the LAV users is unknown but is likely less than 5 years. HAV users demonstrated a diverse range of experience (Fig.1). LAV users were all in the 18-24 age bracket whereas HAV users showed more variation (see Fig.2).

i)

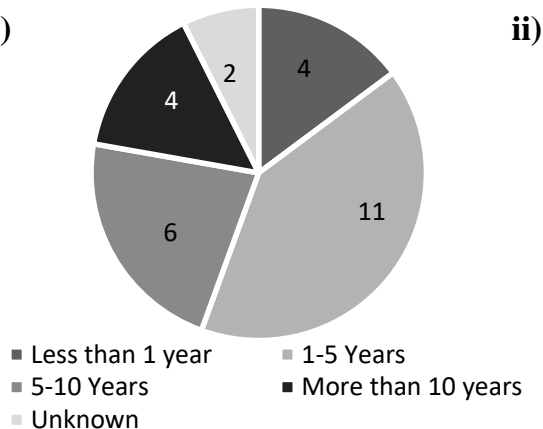

ii)

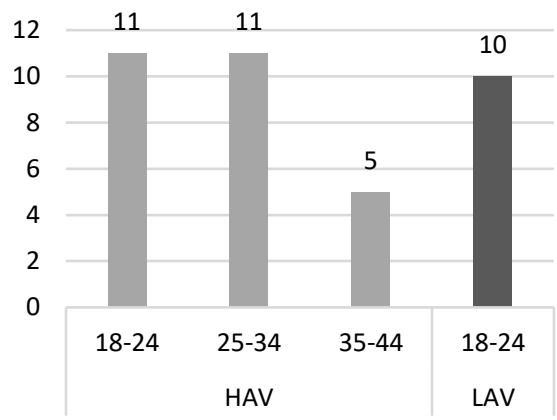

Fig. 1. i) Level of experience indicated by HAV questionnaire respondents. ii) Number of participants in each respective age bracket according to type of vehicle used

\subsection{Questionnaire Design}

Participants assessed the system by responding to a battery of well-established evaluation questionnaires. One part examined the physical aspects of vehicle use, another examined the OCC and the third the WT/TCC.

Physical Ergonomics was assessed using the Dutch Musculoskeletal Questionnaire (DMQ; [18]), the Nordic Musculoskeletal Questionnaire (NMQ) and the Body Part Discomfort Questionnaire (BPDQ [19]). There is supporting research that work intensity correlates with musculoskeletal complaint (MSC) development [20][21] which results in lost-work-time [22]. Resultantly, MSCs can adversely affect the operational capabilities of defence forces. The DMQ analyses musculoskeletal workload using 5-point frequency rating scales (1- often, 5- never). It exploits the relationship between work tasks and MSCs [23] to identify MSC development risk. The original 63-item questionnaire was reduced to 34 -items to remove irrelevant items. For instance, workplace vibration and weather questions were removed as these were outside of the scope of the user's tasks such as 'making short maximal force-exertions'. 
An adapted version of the NMQ was used which consisted of users reporting any MSCs (Yes / No) across nine anatomical areas over the past 12 months, indicating the injury impact and whether they required a GP consult. The BPDQ was used to evaluate user discomfort at different body parts on a scale of 0-4 (0- extremely comfortable, 4 extremely uncomfortable). Additional questions addressed the visibility and space inside the vehicle as these are potential mitigating factors on user performance.

Cognitive Ergonomics was assessed using the Usability, Satisfaction and Ease of Use (USE) questionnaire [24], the Questionnaire of User Interface Satisfaction (QUIS) [25] and the NASA-TLX [26]. The USE measures the subjective usability of a product or service. The 27-items examined four dimensions of usability; usefulness, ease of use, ease of learning and satisfaction using a 5-point agreement rating scale (1strongly disagree, 5- strongly agree). The QUIS assessed the HMI usability in specific contexts across four categories (screen, terminology/system information, learning and system capabilities) on 10-point rating scales. The scale anchors vary depending on the statement but generally, the higher score is a more positive response. The NASA TLX is the most cited self-report based workload measure [27]. User's rate their responses to six subscales (mental, temporal and physical demand, effort, frustration and performance) based on their experience of the task (the scale was adjusted to a 10-point scale for consistency). Additional questions on HMI efficiency in task support were included.

\section{$3 \quad$ Results}

\subsection{Physical Ergonomics}

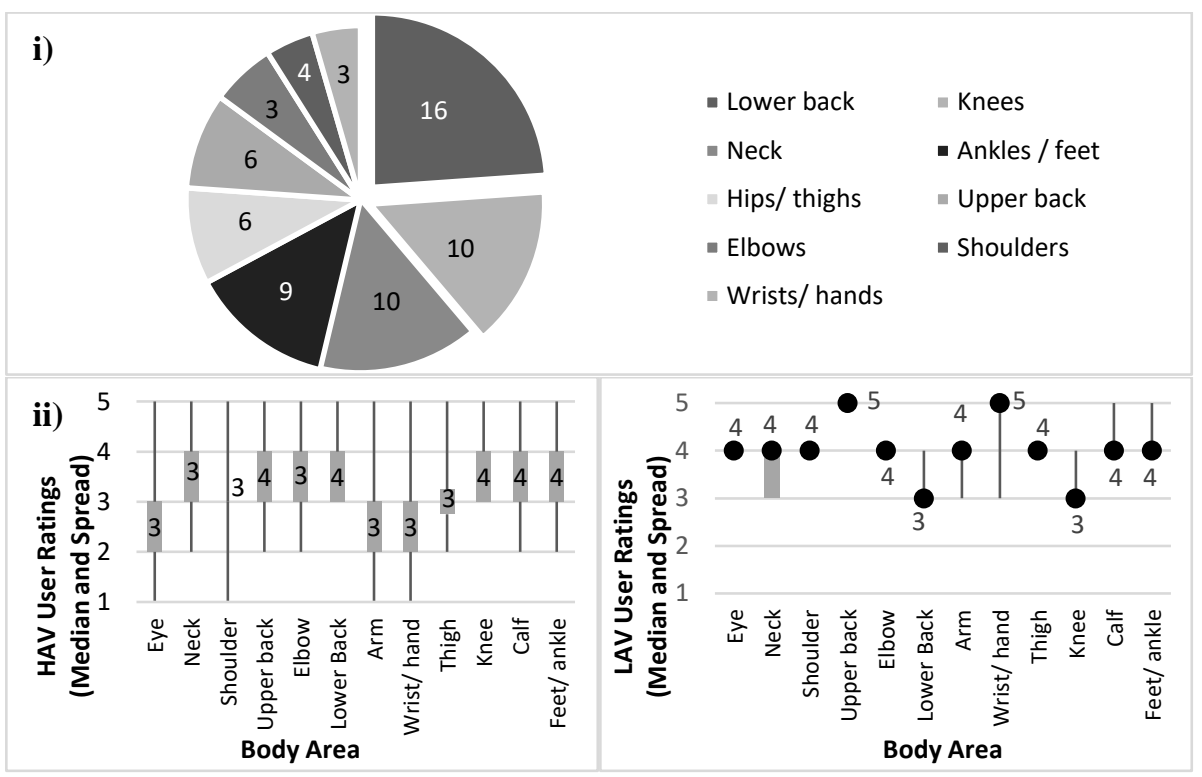

Fig. 2. i) Pie chart showing HAV user MSCs related to a specific body part. ii) HAV and LAV user responses to the BPDQ. A higher score indicates more discomfort. 
Participants completed the NMQ to indicate any MSCs over the past 12 months. NMQ feedback showed that no LAV users had any MSCs in the timeframe. In contrast, HAV users showed a higher incidence of MSCs with 18 of the 27 (66.7\%) reporting a complaint. The most common source of pain was in the lower back followed by knees and neck (Fig. 2i). In line with this, participants also reported the level of comfort they experienced in each area of the body when operating the LAVs and HAVs using the BPDQ (Fig. 2ii). From Fig.2ii, HAV users reported moderate discomfort in their upper and lower back, knees, calves and feet/ankles. The remaining areas were generally acceptable in terms of comfort. In contrast, LAV users reported higher levels of discomfort in the wrists/hands and upper back, corresponding to our knowledge of a smaller workspace associated with the LAV.

DMQ questionnaire results are displayed in Fig. 3. DMQ responses ranged from 42 to $124(24.7 \%$ to $72.9 \%)$ for the HAV and 106 to $115(62.4 \%$ and $67.6 \%)$ for the LAV. Average scores of $49.1 \%$ and $62.7 \%$ in the HAV and LAV reflect moderate MSC risk.

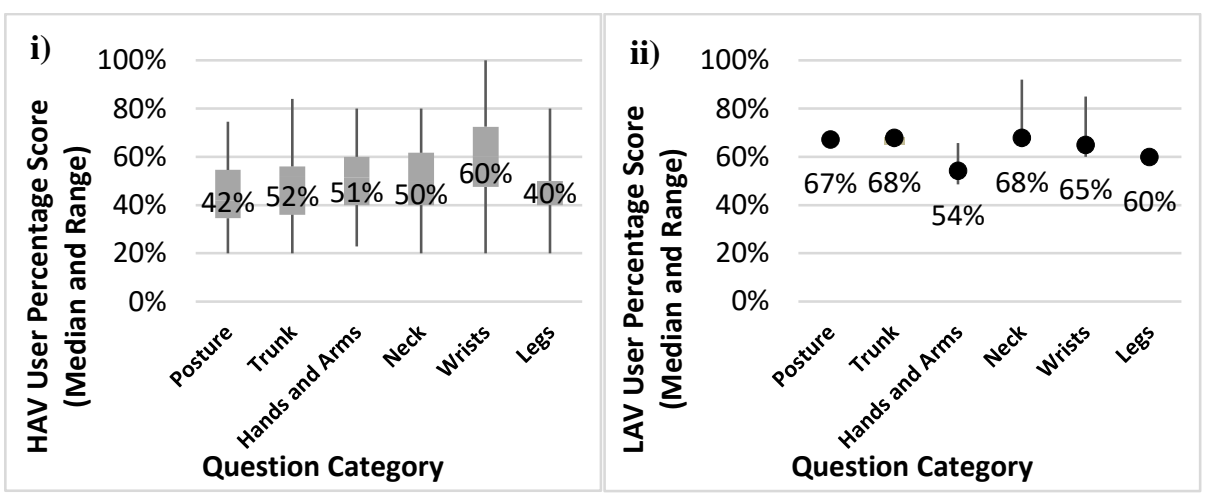

Fig. 3. Box and whisker plots displaying the median and range of i) HAV and ii) LAV user percentage scores for the DMQ body areas. The higher the score the better the physical work environment.

Regarding space, the legroom, headroom and overall space inside the HAV were rated as poor (all items: Mode $\leq 1$ ). 'Ability to reach controls' was acceptable (Mode =4). LAV users were more positive scoring all aspects in the 'Good'/'Very good' range (Mode $=\geq 6$ ). Visually, the ability to survey the environment outside the HAV was rated as moderately poor (Mode $=2$ ) whilst the 'ability to see other team members' was rated acceptable (Mode =4). LAV users again scored more highly, rating the ability to see outside as Good (Mode =6) and the ability to see inside as Very Good (Mode =7).

\subsection{Cognitive Ergonomics}

\section{Commander Console.}

Twenty-six users completed the Commander questionnaire (sixteen HAV; ten LAV). Both HAV and LAV users scored the systems very positively with all subscales receiving above $70 \%$ except for satisfaction which scored above $65 \%$. 


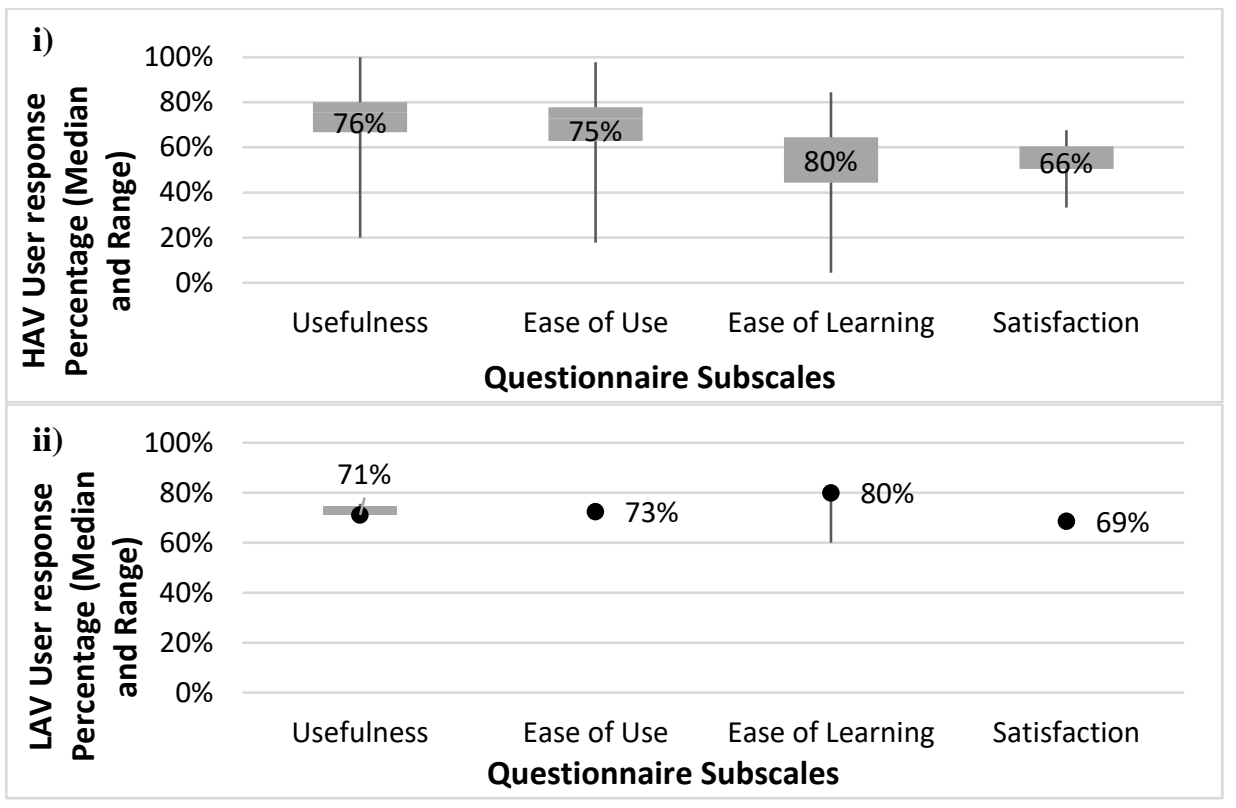

Fig. 4. i) HAV and ii) LAV user percentage scores and response range across the USE Questionnaire subscales. A higher the score reflects a more positive review of the interface.

A full range of responses was recorded across HAV USE individual items, hence the modal response was used to indicate a response consensus. In terms of usefulness, HAV users agreed that the interface was successful in improving their own, their teams and mission activity control (all items: Mode $=4$ ). Conjointly they reported that the interface fulfils its purpose and meets their needs (both items: Mode $=4$ ). Less positively viewed was how streamlined tasks were (Mode $=3$ ). LAV users were also generally very positive about their interfaces, agreeing that the console was successful in improving their own and fulfilled its purpose (all items: Mode $=4$ ). LAV users reported lower ratings for how well the console meets their needs, making the missions easier, controlling vehicle operations and streamlining the process (all items: Mode $=3$ ).

HAV users agreed the interface was easy to use by indicating a low operational effort, lack of expertise required and simplicity (all items: Mode $=4$ ). LAV users reported the system as slightly more complex to use, scoring lower on items such as the effort to use, recovery from errors and the number of steps to complete objectives (all items: Mode $=3$ ). They were more positive about the lack of expertise required and the success of each use (all items: Mode $=4$ ). HAV and LAV users gave modal responses of 3 across most aspects of satisfaction such as frustration to use, satisfaction, pleasantness of use, recommending the system and how the system works, making it the lowest scoring of the four USE subscales.

The QUIS assessed interface usability and design during set-up, surveillance/engagement and overall system operation. Fig. 5 shows the percentage scores on each subscale of the QUIS. Differences between HAV and LAV users were mainly on the 
learning aspect of the interface. HAV users reported learning to set-up as more difficult whereas LAV users reported learning the engagement process as more difficult. Secondly, the screen showed some discrepancies between groups with LAV users reporting layout and information sequence being harder to follow in surveillance and overall.

HAV QUIS set-up responses show that most aspects fall within the 'acceptable' response range, i.e. modal values between 4 and 6 . The interface appears to excel in is that ability to explore features (Mode $=9$, Range $=3-9$ ). The range of responses from HAV users was much larger than LAV users' with most responses ranging from 3 to 9. LAV users responded positively on a range of items including organisation of information, progress updates, system speed and ability to correct mistakes (all items Mode= 6 , Range $=6-9)$. Only the system awkwardness received a score of '4' $($ Range $=2-4)$.

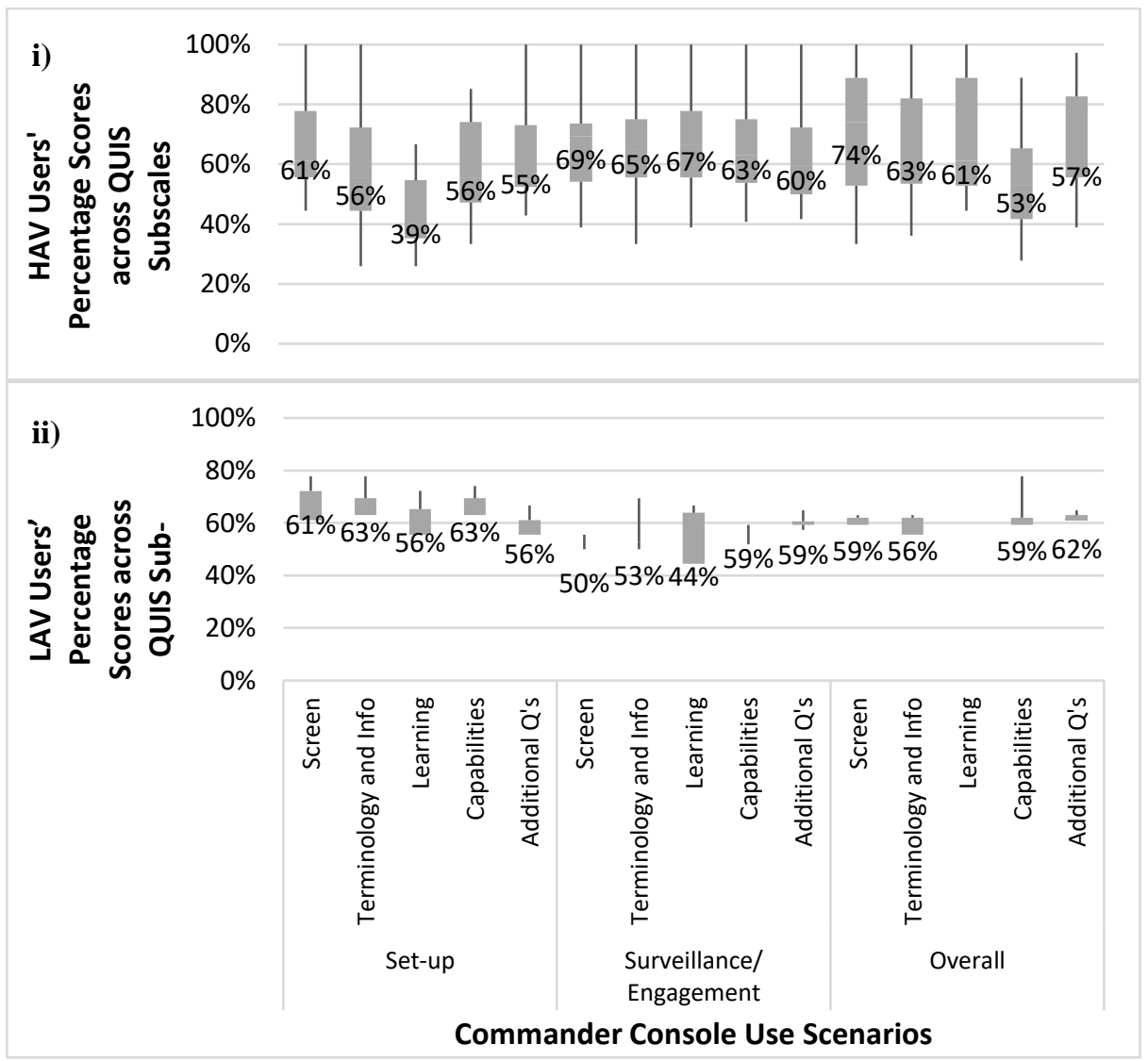

Fig. 5. Box and whisker plot showing the median and range of i) HAV and ii) LAV user responses to the Tactical Commander Console (TCC) and Weapon Terminal (WT) respectively

Regarding surveillance/engagement, HAV scores indicate that again the interface serves its purpose to an acceptable level. However, in this instance more scales showed more agreeable responses to items such as the task sequences being easy to follow, the 
task being straightforward and the task being easy to perform (all items: Mode $=7$, Range $=1-9)$. The least positive response was for the understanding of error messages (Mode $=4$, Range $=2-9$ ). Again, there was a broad range of responses. LAV users again responded mostly along the 'acceptable' segment of the scale (Mode=4-6), with only the system 'awkwardness' being less positively reviewed (Mode $=4$, Range $=2-4$ ).

Lastly, overall Commander Console feedback followed the previous QUIS trends, showing both LAV and HAV users were generally neutral about the interface. The only aspects that HAV users rated more positively was the ability to read characters on the screen (Mode=7, Range=3-9) and that the position of messages was consistent (Mode $=8$, Range $=3-9$ ). Again, LAV users were very consistent in scoring the system between 4 and 6 on the scale. Only one item received a score of ' 4 ', being the item 'procedures can be completed by all levels of users (Mode $=4$, Range $=4-6$ )

\section{Operator Console.}

Eighteen HAV and ten LAV users completed the Operator questionnaire comprising the USE and QUIS questionnaires. Overall, HAV and LAV responses were less positive across all USE subscales for the Operator's console compared to the Commander's console. Fig 6 shows the respective subscale scores.

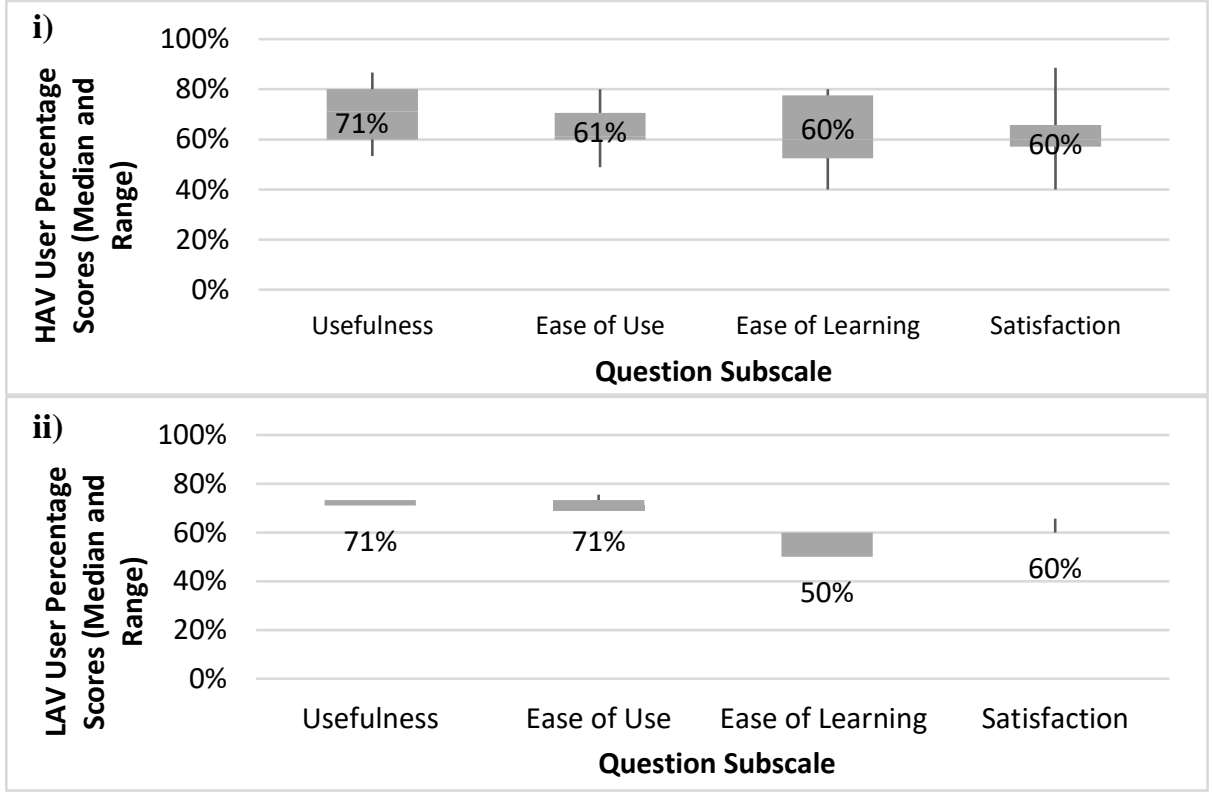

Fig. 6. Box and whisker plot showing the median and range of responses given by i) HAV and ii) LAV users in the USE questionnaire regarding the Operator's Control Console (OCC)

HAV users responded positively on usefulness items including 'provides good control over mission objectives', meets user needs and fulfils its purpose and is simple and easy to use (all items: Mode =4). All other elements of the USE showed a modal score of 3, 
meaning there is room for improvement on aspects such as the steps required to complete procedures and how effective the system is in supporting the crew and user objectives. LAV users responded 'Disagree' with the statements 'I can recover from mistakes quickly and easily' and 'I easily remember how to use it' and 'It is pleasant to use' (all items: Mode $=2$ ) indicating there are some operational issues for LAV users.

The QUIS for OCC set-up showed that for LAV users the organization of information was slightly difficult to follow and the system was slightly awkward to use (all items: Mode $=4$, Range $=3-5$ ) but that the interface in its current state is effective in supporting set-up. All other items scored 5 or 6 indicating the set-up process was effectively supported by the interface. HAV users had a wide range of responses. The users scored the system less favorably on several questions including whether error messages were helpful, the relevance of information on the interface and how streamlined the process is (all items: Mode $=4$, Range 0-7). More favorable aspects included the organization of information, following the task sequence and the straightforwardness of engagement (all items Mode $=6$, Range $=0-7$ ).

Regarding engagement, LAV users report the system to be somewhat awkward to use (Mode $=4$, Range $=3-5$ ). On the other hand, LAV users rate more highly that the information is relevant, there is good control over the task and it is effective in performing the mission (all items: Mode $=6$, Range $=5-6$ ). HAV users reported that error messages were somewhat unhelpful, mistakes are quite difficult to correct and that some experience is required to operate the system (all items: Mode $=4$, Range $=0-7$ ). They responded positively towards the systems' ability to support engagement, the speed at which it is completed and the organization of information (all items: Mode $=6$, Range $=$ 0-9). Users responded similarly to the control panel with LAV users reporting slight problems with the speed of task completion, complexity and awkwardness of use (all items: Mode $=4$, Range $=1-6$ ). The panel is, however, good in that controls are appropriate, the number of controls is sufficient and it streamlines the process (all items: Mode $=6$, Range 5-8). HAV users responded positively about the organization of information, panel speed and the control it provides (all items: Mode $=7$, Range $=1-9$ ) but report it to be somewhat awkward to use (Mode $=3$, Range $=0-7$ ). The overall system was again quite neutrally reviewed with scores ranging from 4 to 6 (on mode) for HAV and LAV users.

\section{Workload.}

Lastly, participants rated the workload associated with each procedure on their respective interfaces (Fig.8). The results show that the Commander's WT in the LAV received consistently higher workload scores on all subscales expect performance compared to the TCC in the HAV. This would indicate that the LAV users experience more difficulty in operating the console.

Regarding the OCC, the results were more comparable across demand and performance subscales. Generally, similar levels of mental workload were observed between the groups but LAV users expressed more effort and frustration with procedures using the OCC. 


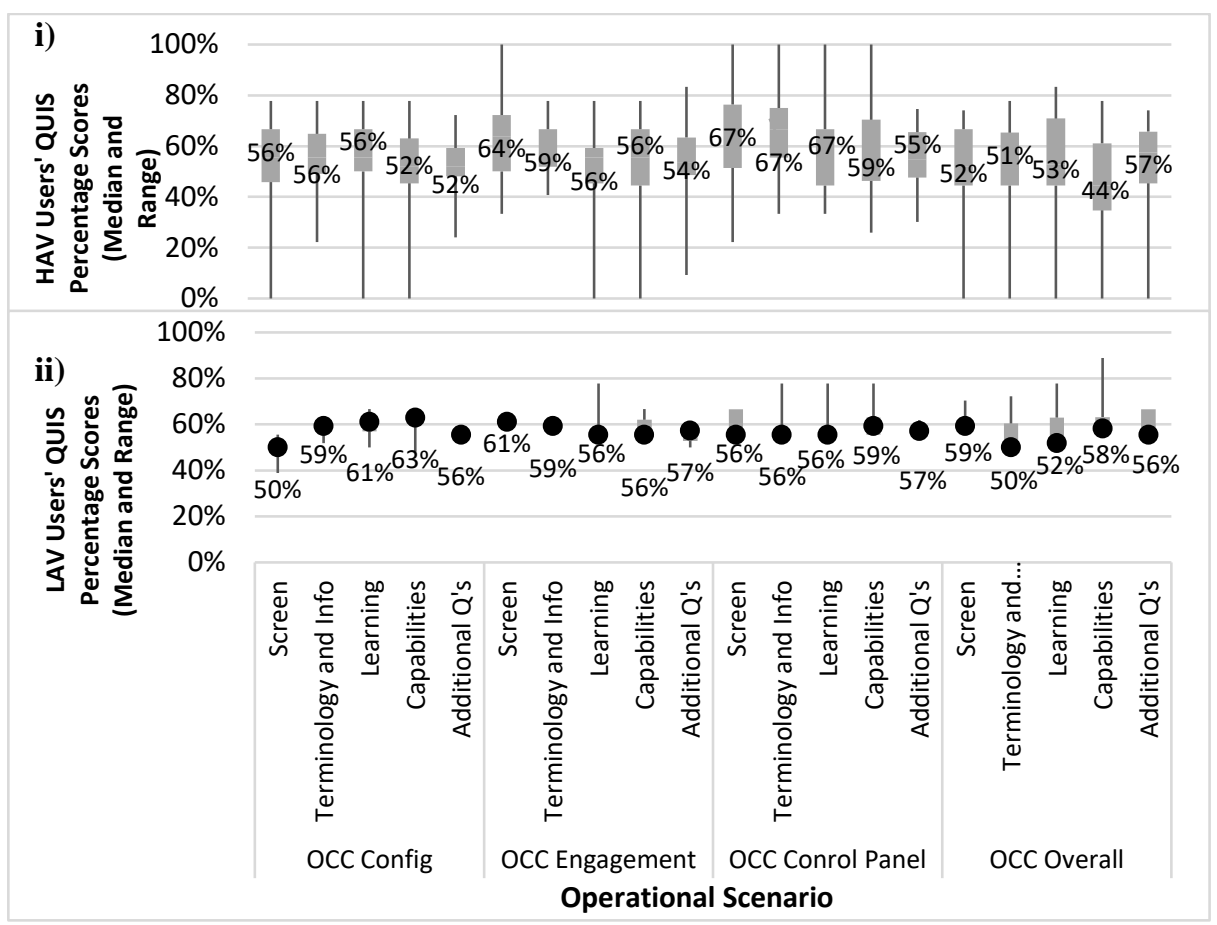

Fig. 7. Box and whisker plot showing the median and range of i) HAV and ii) LAV user responses to the Operator Control Console (OCC).

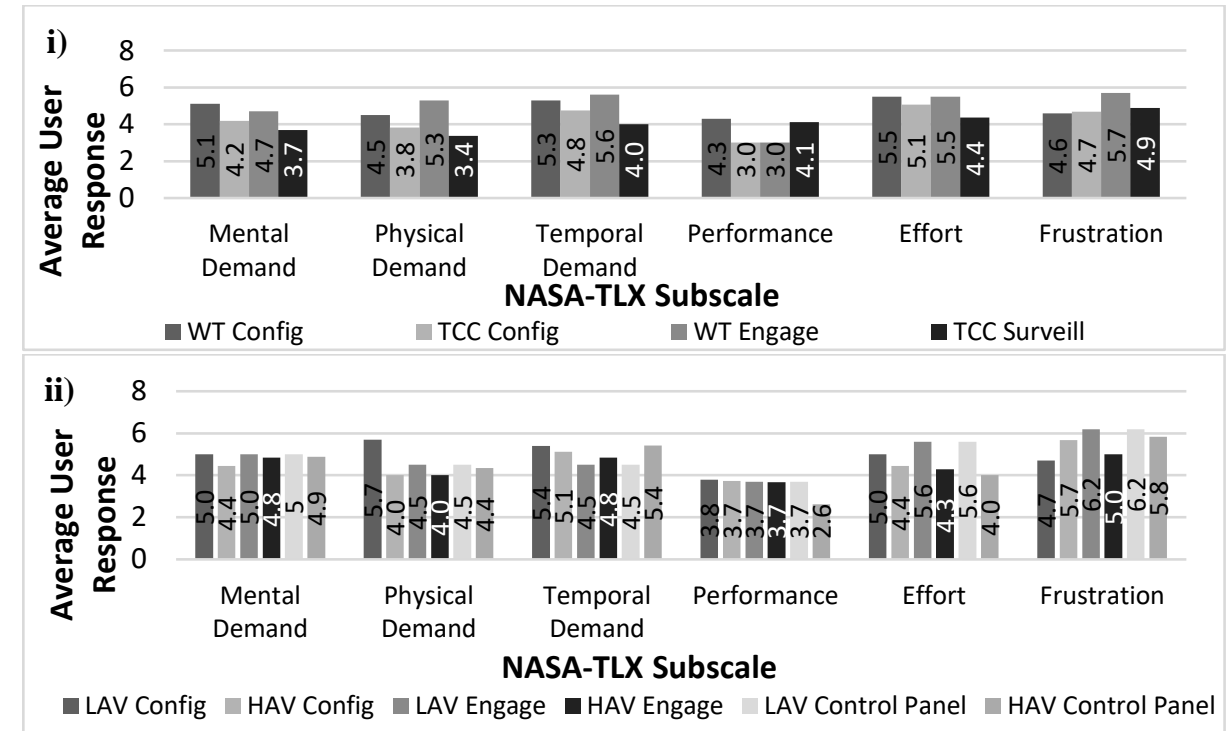

Fig. 8. i) Chart showing the average NASA-TLX scores for each operation on the Commander's respective interface. ii) NASA-TLX scores for operations on the Operator's console 


\section{Discussion}

Overall, the results of the subjective feedback for the LAV and HAV vehicles are generally positive. Physical ergonomics evaluation showed some issues with space and some risk of developing MSCs with prolonged vehicle use. Regarding the weapon system itself, users tended to respond in the mid-range of most items, indicating the system is fit for its purpose but there is room for improvement. The sections below are supplemented with feedback obtained from an HAV user focus group.

\subsection{Physical Ergonomics}

Regarding physical ergonomics, HAV users reported the physical space available to them as an issue. This corresponds to the current understanding of the system limitations; the interior of the vehicle is cramped and considering the amount of equipment and protective gear users will be wearing the issue is further exacerbated. This is a common feature across military vehicles as evidenced in a report that showed a primary complaint from the Marine vehicle operators was a lack of space to stow personal items during missions, such as rucksacks, rations and spare ammunition [28]. A further study [29], noted that operations in military vehicles are characterised by confined-space workstations which require sustained, relatively static postures, leading to a gradual increase in discomfort levels and fatigue, and a decrease in task performance (speed, accuracy and task completion). Combining the lack of space with the resultant levels of fatigue that HAV and LAV users both report, makes optimal performance difficult to achieve. Considering the impact that discomfort can have, as well as the importance of operator performance in a military context, future workspace designs should consider carefully the organisation and placement of equipment to maximise operator space. This agrees with HAV user consensus in the focus group where users highlighted the future system would likely be more spacious and more in line with the LAV design.

\subsection{USE and QUIS Questionnaire Findings}

The key difference between the LAV and HAV used in this study was the Commander's operating console. In the LAV, the console receives orders from higher command and the Commander decides whether the vehicle can/cannot follow the order (e.g. based on vehicle status, orientation etc.). In the HAV, the Commander takes on a larger role. Targets are not identified by higher command but are detected, identified and handled by the crew in the vehicle. The TCC (HAV) contains detailed maps of the environment, surveillance options, imaging, IFF (interrogation friend or foe), target tracking and cueing. The WT (LAV) is more simplistic in its function and layout, displaying only a rudimentary target tracking output and engagement status.

What was surprising in our results was the similarity of feedback for the different consoles. User feedback on the USE questionnaire showed very similar ratings across the usefulness, ease of use, ease of learning and user satisfaction across both interfaces (Fig. 4). Here, a user's experience may have a role. A study [30] showed that users with more experience of a product tended to provide higher, more favourable usability scores 
over those with limited or no experience. The more experienced HAV operators may have reported the interface more favourably than the inexperienced LAV users; potentially explaining why the USE feedback for the HAV was more positive than expected.

In the LAV, USE questionnaire users scored ' 3 ' many times, indicating neither agree nor disagree indicating room for improvement in future interfaces. For both HAV and LAV users, streamlining procedures was highlighted concurrently with interface-related frustration. Research has shown that negative emotional states such as frustration may lead to aggressive behaviors and negatively impact the comfort of drivers [31]. Frustration can be a result of the interface diverging from the user's needs, leading to increased dissatisfaction [32]. This can often compliment and relate to how streamlined the process is, i.e. making the system efficient, maximizing its speed and simplicity.

Feedback on the design and layout of the OCC was less positive for HAV users than the TCC. Users generally responded around ' 3 ' on the scale indicating neither agree nor disagree with the statements. LAV users reported similarly, only identifying their ability to recover from mistakes, their ability to remember how to use it and the pleasantness of the system, as areas that should be improved. These aspects could also be related to overseas users speaking a different native tongue. The LAV operators speak different languages and have different cultural backgrounds but must learn to use the English-based interface. Users relying on their knowledge a second language will complicate their model of the system and will make remembering system use and correcting mistakes more difficult than those utilizing their primary language. A recent paper highlighted that military personnel are now required to develop 1. Breadth (an expanded set of competencies); 2. Depth (higher levels of skill, e.g., critical thinking, anticipation) and 3. Velocity (gain competencies more efficiently and maintain their relevance in an ever-changing environment) [33]. The language barrier LAV users experience may mean that their method of learning may not easily facilitate three aforementioned requirements of military learning. Furthermore, the focus on cognitive readiness in military units and personnel means that mission-essential tasks need to be tied to the underlying cognitive capabilities to perform missions successfully [34]. This means there is an increased need to develop more context-free, transferable instructional abilities [34].

For the QUIS the LAV and HAV Commander consoles received similar feedback with users generally reporting the interface enabled them to complete the tasks relatively well. Regarding the TCC set-up, users indicated some difficulty with inputting information, correcting mistakes and again with how streamlined the procedure is (scores in the lower 50\% of the scale). The engagement process QUIS only highlighted the error messages as a potential cause for concern in this respect, with most of the other items on the scale receiving positive feedback, particularly the task sequences being easy to follow and the ease of performing the task. The WT users reported some issue with the set-up being awkward to perform, which was consistent with what the users reported for the engagement procedure. As the LAV users are non-English speaking perhaps this could be down to a lack of understanding of the system information, leading to confusion regarding what the system requires of them.

On speaking with a training representative at Thales UK, it was clear that language is a significant barrier to training LAV users. LAV users adopt a mimicking approach to learning without any deep understanding or comprehension of the task/ procedure. 
Similar to skill-based approaches, the users are attempting to rapidly acquire the ability to perform the task but when faced with abnormal scenarios this knowledge may fail and impact their ability to adapt. Whilst practicing to perform the specific task they develop routine expertise, but this does not necessarily translate to adaptive expertise i.e. the ability to adapt to non-routine scenarios [35][36]. The WT NASA-TLX scores could corroborate this suggestion as scores for both configuration and engagement indicate all aspects of demand, as well as effort, was higher for WT users. This suggests that WT users struggle more with its operation than their TCC counterparts.

Regarding Operator QUIS feedback, again the two vehicles' OCC received similar feedback with users generally reporting the interface enabled them to complete the tasks well. LAV users reported some problems with the organization of information and the awkwardness of the system during set-up. HAV users found error messages somewhat unhelpful, they expressed some concern over information relevance and the streamlining of information. These issues reflect some of what was found in the Commanders QUIS. Again, for engagement LAV users reported some level of awkwardness and the HAV users reported some problems with how streamlined the procedure was, the error messages and the ease of correcting mistakes. Interestingly, HAV users report more expertise is required for the OCC than the TCC.

Lastly, when operating the control panel again the concept of awkwardness was apparent in both LAV and HAV users. In this case, the physical awkwardness of operating the panel could be, in part, due to the lack of space inside the respective LAV and HAV vehicles. The OCC control panel has two handles that extend into the legroom of the user and these are responsible for the firing sequence of the weapon system. These were reported as an issue from the qualitative feedback section of the questionnaire, where users suggested removing at least one of the handles. NASA-TLX scores for workload in the OCC reflect the same as above, LAV users scored higher for overall workload than their HAV counterparts. HAV users scored each procedure very similarly on average. Both LAV and HAV user's score frustration the highest dimension on average. What is promising is that although frustration and demand is high, users of both the LAV and HAV report their performance as very good, indicating that they are capable of coping with the workload to perform mission tasks effectively, this coincides with the HAV focus group reporting that generally the system itself is good but it is the vehicle the system is mounted inside that is causing most of the usage issues.

\subsection{Design Implications and AI Potential}

Questionnaire feedback suggests some design implications for future weapons systems. Firstly, utilizing space available more effectively as users in this study highlighted issues with space when operating inside the AVs. Inefficient use of space could be placing users at a higher risk of MSCs, reducing their effectiveness in the battlefield and during missions. Users noted that the handles of the OCC were obtrusive and effected comfort and posture inside the vehicle. Unfortunately, comments from the focus group indicated that, due to design and weapon system safety considerations, there is little room for adjustment in these aspects. As such, basing the weapons platform on a dif- 
ferent vehicle is a more practical solution. Additionally, improving interface accessibility (particularly for LAV users) such as improving the organization of information, deciphering task progress and system complexity could improve usability. Facilitating better training procedures and nurturing adaptive expertise could be an effective approach to these issues. Using better symbology and adapting to local terminology could improve the interface for overseas users. Lastly, improving how streamlined the procedures are for HAV users in the form of removing irrelevant information surrounding task performance, reducing the complexity of the information displayed and improving the organization of the information would be beneficial. This could contribute to workload reduction as well, reducing distraction by making key information easier to locate.

With these in mind, advances in recognition, classification and decision-making algorithms (collectively now referred to as AI) could be embedded in the display and control software of a future weapon system with the aim of reducing user workload and aiding user decision-making. Whilst the level of Operator and Commander workload is comparable between the two vehicles, it is the Commander's role that is essential in determining vehicle actions. Aside from removing the Operator and automating the firing sequence (which has significant ethical and moral considerations; see [37]), improving the layout and design of the interface is the most viable way of improving the operator's workload. This was also agreed with the HAV user focus group.

It seems more likely that AI in the LAV WT or the HAV TCC would be more advantageous to improve Commanders' ability to decide on the correct course of action. In the LAV, AI could assume a large proportion of the Commander's activities including deciding if the vehicle can engage a target by assessing weapon system status (such as missiles available, whether it is in range and in the right orientation). The Commander would be tasked with monitoring the $\mathrm{AI}$ to ensure correct decisions are made based on the AI output. In the HAV, AI could acquire and present some of the decisionvital information for the Commander to aid them in their decision-making tasks. An AI system could be adopted that would aid in identifying a target; such as whether a target is located where it should be and the type of target. Ultimately the Commander will still be in control of the final decision which is a property the HAV focus group insisted was vital in future $\mathrm{AI}$ integration. Avoiding the system making decisions or causing a confirmation bias was reported as essential in ensuring responsible and carefully considered decisions are made. This form of AI could save Commanders vital seconds in their decision-making, increase efficiency efficient and reduce the number of errors.

\subsection{Limitations}

One of the main limitations of this study is the subjectivity of the questions and response scales. This is a common problem across questionnaires [38][39][40]. Terminology can be interpreted differently by individuals. In addition, this study had the added complexity of English being a secondary language, increasing the chances of miscommunication. Moreover, our users in this study had only experienced the use of one of the systems; results may have been more useful had users experienced both the systems, enabling a better comparison between the two vehicles. This may also have helped with the issue of subjectivity as user responses would have been more readily 
compared between questionnaires. Furthermore, the consistency of LAV user responses could place some doubt over the generalization of the results. Culturally, overseas users are less known for complaining. For instance, Chinese and American politeness behaviors are vastly different, meaning their approaches to complaining may be vastly different [41]. The same rule could apply to the users in this study where, culturally, the LAV users may be less inclined to review their vehicles in any negative way; meaning their results are more positive than their true perspectives. Lastly, user experience levels were unbalanced between the two samples, with LAV users being younger and less experienced. HAV Commanders with more experience tended to score the vehicles more positively in terms of physical ergonomics, whereas users in the middle age group (25-34) scored the interface more favorably across the QUIS. The same is true of the HAV Operators, the 25-34 age group scored the interface more favorably than the younger and older age groups. Workload scoring between the levels of experience was, however, consistent between age groups.

\subsection{Conclusion}

This study aimed to utilise a questionnaire battery to assess user opinions on the HMI design of two currently used armoured vehicles. Despite the limitations, the results provided valuable insight into the efficiency of the system and prompted avenues for future weapons system improvement. Overall, the study successfully highlighted the concerns users had when using the weapon system and identified how future vehicle development could integrate AI technology. The key conclusions from the study are:

- Users' posture and physical work environment showed a moderate MSC risk. Future system development needs to maximize available space and consider user-system interactions more carefully to promote long-term health and user performance.

- Responses showed users were generally satisfied with the current system with only some refinement suggestions. These include streamlining the procedures and information provided to improve overall system usability and increase user satisfaction which could also aid with the issues reported by LAV users.

- LAV reported higher workload, more difficulty in learning and remembering the system functions and more frustration with system operation. This can be partly attributed to a language barrier in system training and design. Future systems (when designed for a global market) should thereby carefully consider this earlier in the design process, facilitating flexibility as much as possible e.g. by representing functions and information in language-neutral ways such as through symbols.

- Integrating an AI component into the weapon system that would support the Commander's decision-making process appears to be the most beneficial use of the technology. This would save vital seconds in decision-making time, increase efficiency and reduce the number of errors. This approach would also be more accepted by users as it aids the decision-making process whilst also avoiding confirmation bias and coercing/ leading users into making a specific decision. 


\section{References}

[1] C. C. Krulak, "The strategic corporal: Leadership in the three block war.," Marine Corps Gazette, vol. 83, no. 1, pp. 18-22, 1999.

[2] T. C. Yean, M. S. Hong, and V. Yew, "Fighting Vehicle Technology," 2013.

[3] K. E. Friedl, S. J. Grate, S. P. Proctor, J. W. Ness, B. J. Lukey, and R. L. Kane, "Army research needs for automated neuropsychological tests: monitoring soldier health and performance status.," Arch Clin Neuropsychol, vol. 22 Suppl 1, pp. S7-14, 2007.

[4] L. Martinez-Lopez, "Cognitive performance in operational environments." Aviation, space, and environmental medicine, vol. 76, no. 7, pp. C2-C3, 2005.

[5] M. M. Thompson and D. R. McCreary, "Enhancing mental readiness in military personnel.," in Human Dimensions in Military Operations - Military Leaders' Strategies for Addressing Stress and Psychological Support, 2006.

[6] M. L. Thomas and M. B. Russo, "Neurocognitive monitors: toward the prevention of cognitive performance decrements and catastrophic failures in the operational environment." Aviation, space, and environmental medicine, 78 (5), pp. B144-B152, 2007.

[7] R. A. Grier, "Military cognitive readiness at the operational and strategic levels: A theoretical model for measurement development." Journal of Cognitive Engineering and Decision Making, vol. 6, no. 4, pp. 358-392, 2012.

[8] L. Giraudet, J.-P. Imbert, M. Bérenger, S. Tremblay, and M. Causse, "The neuroergonomic evaluation of human machine interface design in air traffic control using behavioral and EEG/ERP measures," Behavioural brain research, vol. 294, pp. 246-253, 2015.

[9] V. Santangelo, "Forced to remember: when memory is biased by salient information," Behavioural brain research, vol. 283, pp. 1-10, 2015.

[10] E. W. Martin, "Artificial intelligence and robotics for military systems." Washington DC, 1984.

[11] C. Gay, B. Horowitz, J. Elshaw, P. Bobko, and I. Kim, "Operator Suspicion and Human-Machine Team Performance under Mission Scenarios of Unmanned Ground Vehicle Operation." IEEE Access., vol. 7, pp. 36371-36379, 2019.

[12] S. P. P. Zieba and F. Vanderhaegen, "Using adjustable autonomy and human-machine cooperation to make a human-machine system resilient-Application to a ground robotic system." Information Sciences, vol. 181, no. 3, pp. 379-397, 2011.

[13] L. A. W. A. J. Bush and B. C. Williams, "Risk-based sensing in support of adjustable autonomy." in IEEE Aerospace Conference, 2012, pp. 1-18.

[14] A. Incose, "A world in motion: systems engineering vision 2025." in International Council on Systems Engineering., 2014.

[15] L. Carver and M. Turoff, "The human and computer as a team in emergency management information systems." CACM, vol. 50, no. 3, pp. 33-38., 2007.

[16] R. Parasuraman and P. A. Hancock, "Mitigating the adverse effects of workload, stress, and fatigue with adaptive automation." 2008, pp. 45-57.

[17] Z. Gibson, J. Butterfield, and A. Marzano, "User-centered design criteria in next generation vehicle consoles," Procedia CIRP, vol. 55, pp. 260-265, 2016.

[18] V. H. Hildebrandt, P. M. Bongers, F. J. van Dijk, H. C. Kemper, and J. Dul, "Dutch Musculoskeletal Questionnaire: description and basic qualities.” Ergonomics, vol. 44, no. 12 , pp. $1038-1055,2001$

[19] E. N. Corlett and R. P. Bishop, “A technique for assessing postural discomfort," Ergonomics, vol. 19, no. 2, pp. 175-182, 1976.

[20] G. J. Engelbrecht, L. T. de Beer, and W. B. Schaufeli, "The relationships between work intensity, workaholism, burnout, and self-reported musculoskeletal complaints," Human Factors and Ergonomics in Manufacturing \& Service Industries, 2019. 
[21] A. Halvarsson, I. Hagman, M. Tegern, L. Broman, and H. Larsson, "Self-reported musculoskeletal complaints and injuries and exposure of physical workload in Swedish soldiers serving in Afghanistan," PloS one, vol. 13, no. 4, p. e0195548, 2018.

[22] A. Riches, W. Spratford, J. Witchalls, and P. Newman, "A Systematic Review and Meta-Analysis about the Prevalence of Neck Pain in Fast Jet Pilots," Aerospace Medicine and Human Performance, vol. 90, no. 10, pp. 882-890, 2019.

[23] J. Dul, N. J. Delleman, and V. H. Hildebrandt, "Posture and movement analysis in ergonomics: principles and research,” in Proceedings of the Symposium 'Biolocomotion: A Century of Research Using Moving Pictures, 1, Cappozzo, A., and Marchetti, M., and Tosi, V., Ed. Promograph, 1992.

[24] A. M. Lund, "Measuring usability with the USE questionnaire," Usability interface: STC Usability SIG Newsletter, vol. 8, no. 2, pp. 3-6, 2001.

[25] J. P. Chin, V. A. Diehl, and K. L. Norman, "Development of an instrument measuring user satisfaction of the human-computer interface." in Proceedings of the SIGCHI conference on Human factors in computing systems, 1988, pp. 213-218.

[26] S. G. Hart and L. E. Staveland, "Development of NASA-TLX (Task Load Index): Results of empirical and theoretical research." Advances in psychology, 52, pp.139-183, 1988.

[27] R. A. Grier, "How high is high? A meta-analysis of NASA-TLX global workload scores." Proceedings of the Human Factors and Ergonomics Society Annual Meeting, vol. 59, no. 1, pp. 1727-1731, 2015.

[28] B. Paul, "Case Study of GPS Retransmission in Military Ground Vehicles," in Proceedings of the 2011 NDIA Ground Vehicle Systems Engineering and Technology Symposium, 2011.

[29] N. J. Delleman, V. Colaciuri, E. Wiederkehr, and P. J. L. Valk, "Sustained operations in confined-space military vehicles.” Int J Occup Saf Ergon, 14(3), pp. 313-25, 2008.

[30] S. McLellan, A. Muddimer, and S. C. Peres, "The effect of experience on System Usability Scale ratings," Journal of usability studies, vol. 7, no. 2, pp. 56-67, 2012.

[31] A. Löcken, K. Ihme, and A. Unni, "Towards Designing Affect-Aware Systems for Mitigating the Effects of In-Vehicle Frustration," in Proceedings of the 9th International Conference on Automotive User Interfaces and Interactive Vehicular Applications Adjunct, 2017, pp. 88-93.

[32] A. Degani, C. V. Goldman, O. Deutsch, and O. Tsimhoni, "On human-machine relations," Cognition, Technology \& Work, vol. 19, no. 2-3, pp. 211-231, 2017.

[33] S. Schatz, D. Fautua, J. Stodd, and E. Reitz, "The changing face of military learning," in Proceedings of the I/ITSEC, 2015.

[34] J. Fletcher and A. P. Wind, "The evolving definition of cognitive readiness for military operations," in Teaching and measuring cognitive readiness, Springer, 2014, pp. 25-52.

[35] L. Bainbridge, "Difficulties and errors in complex dynamic tasks," Ergonomics, in press. Retrieved August 2018, vol. 24, p. 2002, 1998.

[36] D. Embrey, "Understanding human behaviour and error," Human Reliability Associates, vol. 1, no. 2005, pp. 1-10, 2005.

[37] A. Etzioni and O. Etzioni, "Pros and cons of autonomous weapons systems." Military Review, 2017.

[38] S. Jahedi and F. Méndez, "On the advantages and disadvantages of subjective measures." Journal of Economic Behavior \& Organization, vol. 98, pp. 97-114, 2014

[39] M. Bertrand and S. Mullainathan, "Do people mean what they say? Implications for subjective survey data." American Economic Review, vol. 91, no. 2, pp. 67-72, 2001.

[40] D. A. Redelmeier, J. Katz, and D. Kahneman, "Memories of colonoscopy: a randomized trial." Pain, vol. 104, no. 1-2, pp. 187-194, 2003.

[41] C. Hong and S. Shih, "'You Shouldn't Have Done That!'-A Cross-Cultural Study of Perceptions of Appropriate Complaints.” English Teaching \& Learning, 33, no. 1, 2009 